\title{
TRANSIÇÃO NUTRICIONAL: UMA REVISÃO SOBRE HÁBITOS ALIMENTARES DE ESCOLARES
}

\author{
RICARDO, Aline Juliana - Nutricionista, graduada pela Faculdade de Taquaritinga. \\ PEREIRA, Rita de Cássia Garcia - Docente do curso Nutrição do Centro Universitário de Araraquara - \\ Uniara. E-mail: ritanutry@terra.com.br.
}

\begin{abstract}
RESUMO
O processo de transição nutricional dá-se no Brasil a partir das modificações do padrão alimentar brasileiro, concomitante com modificações também no cenário epidemiológico, gerando um declínio da desnutrição, porém um aumento significativo da obesidade infanto-juvenil em todas as classes socioeconômicas. A idade escolar atende a faixa etária entre os 7 e 10 anos de idade e é caracterizada como um período de crescimento com altas exigências nutricionais, que devem ser supridas com alimentos de qualidade e em quantidade suficientes para evitar problemas futuros de saúde. Para isso, a escola, os professores e os familiares devem responsabilizar-se pela construção do hábito alimentar dessas crianças, por meio de projetos de educação nutricional, atividades relacionadas à boa alimentação e informações condizentes ao mundo real das crianças. O presente artigo tem como objetivo descrever alguns aspectos do perfil alimentar de escolares e sua influência em relação ao aumento da incidência e prevalência de obesidade e comorbidades associadas, bem como identificar o processo de transição nutricional pelo qual o país vem passando.
\end{abstract}

Palavras-chave: Escolares; Hábito alimentar; Transição nutricional.

NUTRITIONAL TRANSITION: A REVIEW OF STUDENTS' EATING HABITS

\begin{abstract}
The nutritional transition process has started in Brazil since the modifications of the standard Brazilian food also concomitant with changes in the epidemiological scenario, resulting in a decline of malnutrition, but in a significant increase in obesity in children and adolescents in all socioeconomic classes. The school age comprises the age group between seven and ten years old and is characterized as a period of growth with high nutritional requirements that must be supplied with quality food and in sufficient quantity to prevent future health problems. For this reason, school, teachers and family members should be responsible for the feeding habits of these children, through nutrition education projects, activities related to good nutrition and consistent information according to the real world of children. The aim of this paper is to describe some aspects of the food profile of students and its influence over the increasing incidence and prevalence of obesity and associated to co-morbidities, as well as to identify the transition process that the country has been experiencing.
\end{abstract}

KEYwORDs: Students; Eating habits; Nutritional transition. 


\section{INTRODUÇÃo}

A idade escolar atende a faixa etária entre os 7 e 10 anos de idade e é caracterizada como um período de crescimento com exigências nutricionais altas. A alimentação nessa idade é um fator ambiental de extrema importância, pois envolve o crescimento e desenvolvimento de todo indivíduo nessa fase da vida (NASSER, 2006).

Cabe salientar que a ingestão calórica deve ser baseada em alimentos saudáveis, que proporcionem os nutrientes essenciais ao bom desempenho das funções de todos os órgãos e sistemas do corpo humano, pois é certo que hábitos alimentares inadequados contribuem para o aumento de deficiências nutricionais.

A escola, professores e familiares são os maiores responsáveis pela construção do hábito alimentar de uma criança; por isso, a escola deve ser entendida como o local de educação que desenvolve ações de promoção da saúde e prevenção de doenças para as crianças que dela se beneficiam (SILVA, 2007), cabendo aos pais estar presentes em projetos de educação nutricional, auxiliando na aprendizagem de seus filhos por meio de atividades e informações condizentes ao mundo real dos mesmos (LUCAS, 2005).

As práticas alimentares são adquiridas durante toda a vida, destacando-se os primeiros anos como um período muito importante para o estabelecimento de hábitos que promoverão a saúde do indivíduo; entretanto, podem também ser um período em que a inadequação alimentar também pode contribuir para a formação de hábitos errôneos (OLIVEIRA et al., 2005).

Nos primeiros anos de vida, uma alimentação adequada é de extrema importância para o crescimento e desenvolvimento da criança, proporcionando ao organismo desempenhar satisfatoriamente suas funções, garantindo, assim, o bom funcionamento do corpo ainda em formação (PHILIPPI; CRUZ; COLUCCI, 2003).

De acordo com o "Guia Alimentar para Crianças menores de 2 anos" (BRASIL, 2002), o aleitamento materno exclusivo nos primeiros meses de vida garante a sobrevivência de muitas crianças, principalmente as que nascem com baixo peso, protegendo-as da morte por diarreia ou infecções respiratórias, e ainda tem a vantagem de possuir um baixo custo, beneficiando assim as famílias mais humildes que não possuem condições de proporcionar aos filhos um aleitamento artificial devido ao seu valor elevado.

Porém, na adolescência, período caracterizado como transição da infância para a vida adulta, os hábitos alimentares costumam se modificar bastante e de forma inadequada, como aumento do consumo de açúcares e gorduras saturadas diariamente e a diminuição da ingestão de alimentos fontes de vitaminas e minerais, presentes principalmente nas frutas, verduras e legumes e, até mesmo, a omissão de refeições como café da manhã e lanches intermediários (RUVIARO; NOVELLO; QUINTILIANO, 2008).

Essa inadequação alimentar acarreta uma deficiência de vários nutrientes essenciais ao desenvolvimento favorável de crianças e adolescentes. No estudo de Spinelli et al. (2003), realizado em creches no município de São Paulo, é relatado maior prejuízo para os nutrientes cálcio, ferro e energia na alimentação de crianças menores de 7 anos e, apesar do consumo proteico adequado, os valores de energia, provenientes de carboidratos e lipídeos, foram de cerca de $30 \%$ e $18 \%$, respectivamente, em relação aos valores recomendados pelo Sociedade Brasileira de Alimentação e Nutrição - SBAN, o que pode justificar a presença de anemia nas crianças estudadas, já que esse aporte proteico deve estar sendo mobilizado para o fornecimento de energia. Outros fatores também referenciados pelos mesmos autores são considerados problemas comuns na infância, tais como a inadequação do consumo de fibras, deficiência de vitamina A, que pode provocar o aparecimento de infecções respiratórias, diarreia e sarampo e baixa ingestão de vitamina $\mathrm{C}$, a qual estimula a absorção do ferro nãoheme em até dez vezes.

Dessa forma, é de fácil entendimento a preocupação de estudiosos da área da saúde em promover hábitos saudáveis desde a infância e conscientizar os pais sobre a importância de tais ações, como demonstra o estudo 
de Oliveira et al. (2005), em que revelam que as crianças tendem a imitar os hábitos alimentares de sua família. Por isso, nos últimos anos têm-se criado vários programas no sentido de incentivar as famílias a introduzir alimentos diferentes e de boa qualidade nutricional na alimentação diária das crianças, a estabelecer horários regulares para as refeições e a selecionar utensílios adequados para cada idade, visando a uma melhor aceitação e degustação dos alimentos. Já os lanches, como refeições intermediárias, devem ser saudáveis.

A alimentação desempenha um importante papel durante todo o ciclo da vida humana. Porém, significativa importância deve ser dada às fases da infância e adolescência, já que as crianças a partir dos 7 anos de idade começam a ter sua independência, revelando assim suas vontades e grande capacidade de escolha por alimentos saudáveis, quando bem orientadas (FLÁVIO, 2006).

A infância é a fase da vida em que o ser humano mais necessita de cuidados, especialmente no que diz respeito aos bons hábitos alimentares, pois é nessa época que os alimentos são descobertos e, de acordo com a forma com que são apresentados, aprende-se a saboreá-los ou a rejeitá-los, e essas primeiras impressões são carregadas para o resto da vida, formando o bom ou mau hábito alimentar do indivíduo. Assim, é fundamental que a sociedade, a escola e a família se conscientizem da importância de ensinar às crianças os benefícios da boa nutrição, para evitar futuros problemas de saúde provenientes da má alimentação (GOMES et al., 2005).

Para Valle e Euclydes (2007), uma alimentação saudável, além de proporcionar prazer, fornece energia e outros nutrientes que o corpo necessita para manter seu bom funcionamento, e os maus hábitos alimentares acarretam problemas de saúde imediatos e também no longo prazo.

De acordo com Rivera e Souza (2006), nos últimos anos a população brasileira tem incorporado hábitos alimentares de países desenvolvidos, tendo como consequência o aumento do consumo de alimentos industrializados, repercutindo na ingestão elevada de lipídeos e carboidratos simples, aumentando a incidência e prevalência da obesidade e comorbidades associadas.

Essa situação se confirma pelos recentes dados apresentados na Pesquisa de Orçamento Familiar POF de 2008/2009, do Instituto Brasileiro de Geografia e Estatística-IBGE, no Brasil, que constatou que uma em cada três crianças com idade entre 5 e 9 anos está com peso acima do recomendado pela Organização Mundial da Saúde-OMS e pelo Ministério da Saúde. Ainda segundo a POF, os jovens de 10 a 19 anos com excesso de peso passaram de 3,7\%, em 1970, para 21,7\% em 2009 (IBGE, 2009).

Para Triches e Giugliani (2005), a promoção de hábitos alimentares mais saudáveis está associada a um efetivo programa de educação nutricional envolvendo crianças na fase escolar, com o intuito de diminuir os índices de sobrepeso e obesidade nessa faixa etária e, consequentemente, melhorar a qualidade de vida na fase adulta.

As necessidades nutricionais de uma criança são definidas com base em seu metabolismo basal, taxa de crescimento e atividades desenvolvidas. A energia da dieta deve ser o suficiente para assegurar o crescimento e evitar que a proteína de reserva seja utilizada para o fornecimento de energia (MASCARENHAS; SANTOS, 2006).

Alimentos ricos em fibras têm sido recomendados na dieta de crianças com vistas a beneficiá-las, evitando o risco de constipação intestinal, obesidade, hipercolesterolemia e também na prevenção do diabetes mellitus. Porém, não devem ser utilizados em grande quantidade, pois, como possuem o poder de retardar o esvaziamento gástrico, podem ocasionar prejuízos à ingestão calórica e baixa biodisponibilidade de alguns micronutrientes como vitaminas e minerais (LEÃ̃); STARLING, 2003).

Os micronutrientes são essenciais ao crescimento e desenvolvimento normais do indivíduo, podendo a sua baixa ingestão causar atraso no crescimento ou doenças de deficiência. Desse grupo, os elementos que geralmente se encontram abaixo do nível normal de ingestão na alimentação das crianças são: cálcio, ferro, 
zinco, vitamina $\mathrm{B}_{6}$ e vitamina A(FLÁVIO, 2006).

A água é essencial ao bom funcionamento do organismo infantil, pois representa entre 75 e $80 \%$ da composição corporal do lactente, e a sua deficiência pode levar à morte em poucos dias. Com o passar do tempo, sua proporção vai diminuindo até atingir à do adulto, entre 55 e $60 \%$ do corpo humano. O fato de as crianças necessitarem de maior aporte hídrico com relação aos adultos está relacionado à ingestão calórica, às perdas insensíveis e à concentração urinária, atingindo o nível dos adultos apenas no final da adolescência (LEÃO; STARLING, 2003).

\section{Alimentação Escolar - Merenda e Cantina}

O Programa Nacional de Alimentação Escolar PNAE surgiu no Brasil em 1954, com o intuito de fornecer alimentação aos escolares matriculados na rede pública, suprindo parcialmente a necessidade nutricional desses alunos (FLÁVIO, 2006).

Desde então, a alimentação escolar passou a ser um direito previsto na "Constituição Federal", utilizada por alunos que frequentam a Educação Infantil e o Ensino Fundamental, sendo caracterizada como uma suplementação alimentar fornecida durante o período de aula, devendo suprir, no mínimo, 15\% das necessidades calóricas diária das crianças (KUREK; BUTZKE, 2006).

Além da suplementação alimentar, o programa visa melhorar o aproveitamento das aulas e inserir uma variedade de alimentos na refeição dessas crianças, auxiliando na formação de bons hábitos alimentares e na promoção da segurança alimentar e nutricional desses escolares (FOME ZERO, 2005).

Segundo Schmitz et al. (2008), a escola deve proporcionar a aplicação de programas de educação em saúde, incluindo educação nutricional, sendo um local para práticas alimentares saudáveis, propiciando uma boa educação alimentar para as crianças. Tais programas devem ser divididos em três áreas de ação: educação para saúde, ambientes saudáveis e serviços de saúde e alimentação, pois os alimentos vendidos em cantinas escolares contêm grandes quantidades de açúcares, gorduras e sal.
Independentemente da forma a ser oferecida, a alimentação no âmbito escolar deve respeitar as preferências alimentares dos alunos, buscando a ampliação de seus hábitos alimentares por meio da inclusão de alimentos regionais, diversificados e de qualidade que o aluno possa comer à mesa, servindose na companhia dos professores, com acesso a pratos, talheres e copos adequados, sempre enfatizando uma alimentação saudável (CECCIM, 1995).

Num estudo realizado em Florianópolis-SC, com adolescentes de escolas públicas e privadas, com o intuito de avaliar a adequação do consumo de energia e macronutrientes, obteve-se o seguinte resultado: $32,1 \%$ dos adolescentes da rede pública e $21,8 \%$ da rede privada apresentaram um consumo energético acima do adequado, e $33,9 \%$ e $39 \%$ dos estudantes das respectivas redes apresentaramalto consumo de lipídios. Em outro estudo, na Bahia, foi detectado que 9,3\% e $4,4 \%$ das crianças matriculadas em escolas da rede pública e privada apresentavam sobrepeso e obesidade, com $6,5 \%$ e $2,7 \%$ e $13,4 \%$ e 7,0 , respectivamente (SANTO; MERCÊS, 2005).

A alimentação escolar equilibrada, realizada por meio de cardápios elaborados e controlados por nutricionistas presentes nas unidades de alimentação escolar, é um instrumento fundamental para a recuperação e manutenção de hábitos alimentares saudáveis e ainda favorece o desenvolvimento dessas crianças nos aspectos físico, intelectual, emocional e social (KUREK; BUTZKE,2006).

\section{Transição Nutricional}

De acordo com Cano et al. (2005), o Brasil vive um momento de transição nutricional, em que são observadas mudanças nos padrões alimentares da população em consequência de modificações na sua dieta decorrentes de mudanças sociais, econômicas e influência da mídia, fazendo com que a obesidade represente um fenômeno de incidência cada vez mais elevada em todas as classes sociais.

A obesidade é definida como o desequilíbrio entre o consumo alimentar e o gasto energético e está associada ao sedentarismo e à inadequação dos 
hábitos alimentares, sendo considerada um dos principais fatores da alta prevalência de doenças crônicas, como as cardiovasculares, a hipertensão e o diabetes mellitus tipo II (COSTA; CORDONI JÚNIOR; MATSUO, 2007).

Estudos realizados em algumas cidades brasileiras mostram que o sobrepeso e a obesidade já atingem $30 \%$ ou mais das crianças e adolescentes, como por exemplo, os resultados encontrados para Recife em que $35 \%$ dos escolares avaliados encaixavam-se nessa classificação e em Santos, onde foram avaliados todos os escolares entre 7 e 10 anos, tanto da rede pública como da privada, em que $15,7 \%$ e $18,0 \%$ apresentavam sobrepeso e obesidade, respectivamente, ficando a escola privada com os maiores índices (OLIVEIRA; FISBERG, 2003).

As mudanças ocorridas na alimentação do brasileiro durante as três últimas décadas, tanto no que se refere à qualidade quanto à quantidade de alimentos consumidos, foram decorrentes do processo de transição nutricional (SANTO; MERCÊS, 2005).

Os hábitos alimentares se modificam muito rapidamente, pois a mídia tem o poder de construí-los e substituí-los, sempre que lhes parecer lucrativo (COSTA; CORDONI JÚNIOR; MATSUO, 2007). Nesse contexto, para reverter o quadro epidêmico em torno da alimentação e doenças provenientes de hábitos alimentares inadequados, o desenvolvimento de trabalhos educativos e assistenciais, envolvendo a comunidade escolar, alunos, pais, professores, serviço de orientação, supervisão, direção, e serviços de saúde, considerando a história de vida da criança, a inserção da família no contexto socioeconômico e político com atendimento integral, deve ser ofertado e inserido nas políticas públicas (SILVA, 2007).

A obesidade quando presente já nas fases da infância e adolescência, apresenta grande possibilidade de predominância na idade adulta, trazendo como consequência riscos a saúde denominados doenças crônicas não transmissíveis, das quais fazem parte a hipertensão arterial, o diabetes mellitus tipo II e as dislipidemias (SANTOS et al., 2004). Há que se considerar que, quanto menor a idade em que a obesidade se manifesta, maiores são as chances de a criança se tornar um adolescente e, provavelmente, um adulto obeso. Outros fatores também são determinantes para o desenvolvimento da obesidade ao longo da vida, como, por exemplo, o desmame precoce, a introdução de alimentos inadequados, fórmulas lácteas preparadas erroneamente, distúrbios de comportamento alimentar e relacionamento familiar conturbado (RIBEIRO et al., 2007).

Segundo Santos et al., (2004), nos últimos 20 anos, em nosso país, a obesidade sofreu um intenso aumento em todas as regiões, afetando tanto adultos como crianças, prevalecendo o maior número de casos nas áreas urbanas de nível socioeconômico mais elevado.

Devido ao aumento expressivo da obesidade em todo o mundo e em todas as faixas etárias, tanto em países desenvolvidos como naqueles em desenvolvimento, a Organização Mundial da Saúde - OMS sentiu-se na obrigação de classificá-la como epidêmica (RIBEIRO et al., 2007), atingindo proporções que a classificam como um grave problema de saúde pública (SANTOS et al., 2004).

$\mathrm{O}$ aumento da obesidade infantil é um dos grandes fatores associados a doenças de ordem psicológica, como depressão e comportamento agressivo, que acabam interferindo no relacionamento social, familiar e acadêmico da criança (RIBEIRO et al., 2007), levando à sua marginalização pela falta de aceitação e apresentando como consequência o isolamento social da criança, que passa a brincar menos por se sentir envergonhada pela sua imagem perante os colegas e come emexcesso para aliviar o sofrimento, fechandose assim o ciclo vicioso da obesidade infantil (CANO et al., 2005).

\section{InfluênCIA da Mídia na FormaÇão do Hábito Alimentar InFANTIL}

A televisão tornou-se o veículo de comunicação mais acessível à população nos últimos tempos, sendo uma importante fonte de entretenimento e educação (ALMEIDA; NASCIMENTO; QUAIOTI, 2002).

$\mathrm{O}$ hábito de assistir à televisão durante um longo tempo leva a criança a se encantar com os produtos 
ali divulgados, na maioria das vezes refrigerantes, doces, fast-food, alimentos engordativos e brinquedos (CRIVELARO et al., 2006).

Diante da televisão uma criança pode desenvolver hábitos alimentares inadequados, já que a preferência infantil por guloseimas é visível. Assim, o fato de assistir à televisão está associado não só à influência da mídia sobre o comportamento alimentar das crianças e adolescentes, como também ao sedentarismo, que tem como consequência o sobrepeso e a obesidade, e que pode permanecer na idade adulta, acarretando comorbidades associadas, como diabetes, hipertensão arterial, dislipidemias, entre outros (FIATES; AMBONI; TEIXEIRA, 2008).

A educação nutricional proporciona a construção coletiva de conhecimentos com integração entre equipe de saúde, escolas e família, tendo como objetivo principal informar pais e alunos sobre os benefícios da correta alimentação e nutrição (BIZZO; LEDER, 2005).

Assim, segundo Flávio (2006), os períodos da vida escolar e adolescência são os melhores momentos para uma educação ativa sobre os temas nutrição e saúde, que devem ser implementados tanto pelas escolas como pela família, tendo as escolas públicas maior vantagem sobre as particulares, por integrar o Programa de Alimentação Escolar, que propicia desde muito cedo a adesão de alunos a uma alimentação adequada às suas necessidades, por meio da merenda oferecida nos intervalos das aulas.

Em todo o mundo, inúmeros programas de educação nutricional estão sendo desenvolvidos, com o intuito de prevenir a população contra doenças crônicas provenientes do excesso de peso, que hoje são apontadas como as principais causas de doenças e mortes na idade adulta. Esses programas beneficiam as crianças com orientações nutricionais adequadas e conscientização de que a mudança para hábitos alimentares mais saudáveis não requer tanto prescrição e proibição, mas sim compreensão (VARGAS; LOBATO, 2007).

Dessa forma, para que a educação nutricional no âmbito escolar cause o impacto positivo esperado, é de fundamental importância a utilização de materiais didáticos audiovisuais que provoquem nos alunos a curiosidade sobre o assunto e os levem a pensar de forma crítica, além de buscar soluções para melhor sua alimentação, sem a necessidade de impor conceitos e sugestões prontas, que pouco efeito exercem sobre a educação dos escolares (BIZZO; LEDER , 2005).

\section{Considerações Finais}

Esta revisão teve por finalidade promover conhecimentos sobre o perfil alimentar de crianças em idade escolar, já que é nessa fase da vida que o indivíduo começa a formar suas preferências alimentares e entra em contato com uma infinita variedade de alimentos, necessitando assim que não somente a escola, mas principalmente a família, direcione essa formação para hábitos mais saudáveis, visando evitar o predomínio de uma alimentação inadequada que certamente permanecerá pela vida toda, visto que o processo de transição nutricional pelo qual o Brasil passa implica diretamente no padrão alimentar da população, gerando consequências negativas tanto para o Sistema de Saúde quanto para a formação de hábitos alimentares inadequados.

\section{REFERÊNCIAS}

ALMEIDA, S. S; NASCIMENTO, P. C. B. D.; QUAIOTI, T. C. B. Quantidade e qualidade dos produtos alimentícios anunciados na televisão brasileira. Rev. Saúde Pública, São Paulo, v. 36, n. 3, p. 353-355, jun. 2002.

BIZZO, M. L. G.; LEDER, L. Educação nutricional nos parâmetros curriculares nacionais para o ensino fundamental. Rev. Nutr., Campinas, v. 18, n. 5, p. 661-667, set./out. 2005.

BRASIL. Ministério da Saúde. Secretaria de Política de Saúde. Organização Pan Americana da Saúde. Guia alimentar para crianças menores de dois anos. Brasília: Organização Pan Americana de Saúde, 2002. 152 p. 
CANO, M. A. T.; PEREIRA, C. H. C.; SILVA, C. C. C.; PIMENTA, J. N.; MARANHA, P. S. Estudo do estado nutricional de crianças na idade escolar na cidade de Franca-SP: uma introdução ao problema.

Revista Eletrônica de Enfermagem, v. 7, n. 02, p. 179-184, 2005.

CECCIM, R. B. A merenda escolar na virada do século - agenciamento pedagógico da cidadania.

Rev. Em Aberto, Brasília, Ano 15, n. 67, p. 63-72, jul./set. 1995.

COSTA, M. C. D.; CORDONI JÚNIOR, L.; MATSUO, T. Hábito alimentar de escolares adolescentes de um município do oeste do Paraná.

Rev. Nutr., Campinas, v. 20, n. 5, p. 461-471, set./ out. 2007.

CRIVELARO, L. P.; SIBINELLI, E. C.; IBARRA, J. A.; SILVA, R. A publicidade na TV e sua influência na obesidade infantil. UNIrevista, v. 1, n. 3, p. 1-7, jul. 2006.

FIATES, G. M. R.; AMBONI, R. D. M. C.; TEIXEIRA, E. Comportamento consumidor, hábitos alimentares e consumo de televisão por escolares de Florianópolis. Rev. Nutr., Campinas, v. 21, n. 1, p. 105-114, 2008.

\section{FLÁVIO, E. F. Alimentação escolar e avaliação} nutricional dos alunos do ensino fundamental das escolas municipais de Lavras, MG. 2006. 293f. Tese (Doutorado em: Ciência dos Alimentos) Universidade Federal de Lavras, Lavras, 2006.

FOME ZERO - Associação de apoio a políticas de segurança alimentar. Manual de gestão eficiente da merenda escolar. São Paulo, 2005.

GOMES, D. M.; BASTOS, K. P. L.; SOUZA, E. C. G.; PAIXÃO, J. A.; ARÊDES, E. M. O papel da escola na formação do bom hábito alimentar.

Revista Científica da faminas - Muriaé, v. 1, n.
1, p. 28, jan/abr. 2005.

INSTITUTO BRASILEIRO DE GEOGRAFIA E ESTATISTICA(IBGE). Disponívelem :. http:// www.ibge.gov.br/home/Acesso em 10/02/2012.

KUREK, M.; BUTZKE, C. M. F. Alimentação escolar saudável para educandos da educação infantil e ensino fundamental. Revista de divulgação técnico-científica do ICPG, v. 3, n. 9, p. 139-144, jul/dez. 2006.

LEÃO, E.; STARLING, A. L. P. Nutrição em pediatria. In: TEIXEIRA NETO, F. Nutrição clínica. Rio de Janeiro: Guanabara Koogan S.A., p. 267-278, .2003.

LUCAS, B. L. Nutrição na infância. In: MAHAN, L. K; ESCOTT-STUMP, S. Krause - alimentos, nutrição e dietoterapia. 11. a ed. São Paulo: Roca, 2005. p. 247-267.

MASCARENHAS, J. M. O.; SANTOS, J. C. Avaliação da composição nutricional dos cardápios e custos da alimentação escolar da rede municipal de conceição do Jacuípe-BA. Sitientibus, Feira de Santana, n.35, p.75-90, jul./dez. 2006.

NASSER, L. A. importância da nutrição, da infância à adolescência. In: FAGIOLI, D.; NASSER, L. A.

Educação nutricional na infância e na adolescência. São Paulo: ECN, 2006. p. 31-40.

OLIVEIRA, C. L.; FISBERG, M. Obesidade na infância e adolescência - uma verdadeira epidemia. Arq. Bras. Endocrinol. Metab., v. 47, n. 2, p. 107-108, abr. 2003.

OLIVEIRA, I. S.; BORGES, L. E.; MOURA, A. L. M. T.; MAGALHÃES, M. E. N. Alimentação saudável em crianças de dois a três anos. ENCONTRO DE EXTENSÃO DAUFMG, 8., Anais... UFMG. Belo Horizonte, 2005. Disponível 
em: < http://www.ufmg.br/proex/arquivos/8Encontro/ Saude_6.pdf > Acesso em 21 dez. 2011.

PHILIPPI, S. T.; CRUZ, A. T. R.; COLUCCI, A. C. A. Pirâmide alimentar para crianças de 2 a 3 anos. Rev. Nutr., Campinas, v. 16, n. 1, p. 5-19, jan./mar. 2003.

RIBEIRO, A. F. A.; RAMOS, R. B.; MORAES, T. M.; FREIRE, S. C. Obesidade infantil: conhecimento dos hábitos alimentares entre escolares de 08 a 10 anos. 2007. Disponível em http://www.inicepg.univap.br/INIC_07/trabalhos/ saude/inic/INICG00262_01C.pdf > Acesso em 10 jan 2012.

RIVERA, F. S. R.; SOUZA, E. M. T. Consumo alimentar de escolares de uma comunidade rural. Comum. Ciênc. Saúde, Brasília, v. 17, n. 2, p. 111119, 2006.

RUVIARO, L.; NOVELLO, D.; QUINTILIANO, D. A. Avaliação do estado nutricional e consumo alimentar de adolescentes matriculados em um colégio público de Guarapuava-PR. Revista SalusGuarapuava, v. 2, n. 1, p. 47-56, jan./jun. 2008.

SANTO, E. E.; MERCÊS, G. Sobrepeso e obesidade infantil: influências dos hábitos alimentares e da prática de atividade física. Rev.

Diálogospossíveis, Ano 4, n. 2, p. 93-105, ago./ dez. 2005.

SANTOS, C. S.; MONTEIRO, N. T. G.; GUACHE, H.; HÜLSE, S. B.; LUNA, M. E. P.; GROSSEMAN, S.; ASSIS, M. A. A.; VASCONCELOS, F. A. G. Produção e difusão de material educativo para orientação nutricional de escolares de 7 a 10 anos do município de Florianópolis. Extensio - Revista Eletrônica de Extensão, n. 1, p. 1-9, 2004.

SCHMITZ, B. A. S.; RECINE, E.; CARDOSO, G.
T.; SILVA, J. R. M.; AMORIM, N. F. A.; BERNARDON, R.; RODRIGUES, M. L. C. F. A escola promovendo hábitos alimentares saudáveis: uma proposta metodológica de capacitação para educadores e donos de cantina escolar. Cad. Saúde Pública, Rio de Janeiro, v. 2, n. 5, p. 312-322, 2008.

SILVA, M. B.; SANTOS, M. A.; BARBOSA, L. C.; COELHO, M. P. Saúde através da orientação nutricional na escola. 2007. Disponível em <http://www.inicepg.univap.br/ trabalhos/INICG00747_01C.doc>. Acesso em 19 dez. 2011.

SPINELLI, M. G. N.; GOULART, R. M. M.; SANTOS, A. L. P.; GUMIERO, L. C.; FARHUD, C. C.; FREITAS, E. B.; DANTAS, L. F. Consumo alimentar de crianças de 6 a 18 meses em creches. Rev. Nutr., Campinas, v. 16, n. 4, p. 409-414, out./dez. 2003.

TRICHES, R. M.; GIUGLIANI, E. R. J.

Obesidade, práticas alimentares e conhecimentos de nutrição em escolares. Rev. Saúde Pública, v. 39, n. 4, p. 541-547, 2005.

VALLE, J. M. N.; EUCLYDES, M. P. A formação dos hábitos alimentares na infância: uma revisão de alguns aspectos abordados na literatura nos últimos dez anos. 2007. Disponível em < http:// bvsms.saude.gov.br/bvs/periodicos/ revista_APS_v10n1.pdf >. Acesso em: 30 jan. 2012.

VARGAS, V. S.; LOBATO, R. C. O desenvolvimento de práticas alimentares saudáveis: uma estratégia de educação nutricional no ensino fundamental. Vita et Sanitas, Trindade-GO, v. 1, n. 01, p. 24-33, 2007.

RECEBIDO EM 21/3/2012

ACEITO EM 29/10/2012 